\title{
A UTILIZAÇÃO DA PROGESTERONA NATURAL MICRONIZADA EM GESTAÇÕES GEMELARES NA PREVENÇÃO DO TRABALHO DE PARTO PREMATURO
}

\author{
USE OF NATURAL MICRONIZED PROGESTERONE IN THE \\ PREVENTION OF PRETERM LABOR IN TWIN PREGNANCIES
}

\author{
Juliana Barroso Zimmermmann ${ }^{1}$, Adrianne Maria Berno de Rezende Duarte ${ }^{2}$, \\ Alexander Cangussu Silva², Clarissa Rocha Panconi Piccinini², \\ Fernanda Souza Sampaio ${ }^{3}$, Érica Condé Marques Oliveira ${ }^{3}$, \\ Camila Silva Bernardes ${ }^{3}$, Thamires Teixeira ${ }^{3}$
}

Clin Biomed Res. 2015;35(3):154-158

1 Departamento Materno Infantil, Faculdade de Medicina, Universidade Federal de Juiz de Fora (UFJF). Juiz de Fora, MG, Brasil.

2 Faculdade de Medicina, Universidade Federal de Juiz de Fora (UFJF). Juiz de Fora, MG, Brasil.

3 Hospital Universitário, Universidade Federal de Juiz de Fora (UFJF). Juiz de Fora, MG, Brasil.

Autor correspondente: Juliana Barroso Zimmermmann E-mail: julianabz@uol.com.br Faculdade de Medicina

Av. Eugênio do Nascimento, $s / n$. 36038-330, Juiz de Fora, MG, Brasil.

\section{RESUMO}

Com o objetivo de determinar a eficácia da progesterona natural micronizada em gestações gemelares para a profilaxia do trabalho de parto prematuro, foi avaliada uma coorte histórica de 43 mulheres com gestações gemelares. As gestantes foram divididas em dois grupos: usuárias da progesterona (Grupo 1) e não usuárias da progesterona (Grupo 2). O medicamento foi administrado na dosagem de $200 \mathrm{mcg}$ por via vaginal, a partir de 20 semanas de gestação. Das 43 gestações estudadas, 20 foram tratadas com a progesterona. Não houve associação entre uso da progesterona e época do parto, de forma que o uso da progesterona natural micronizada não impediu o parto prematuro $(p=0,87)$. Portanto, a progesterona natural micronizada não reduziu a frequência do trabalho de parto prematuro em gestações gemelares.

Palavras-chave: Gestação gemelar; progesterona

\section{ABSTRACT}

A historical cohort of 43 women with twin pregnancies were assessed to determine the effectiveness of natural micronized progesterone in twin pregnancies for the prophylaxis of preterm labor. The women were divided into two groups: progesterone users (Group 1) and non-users of progesterone (Group 2). The drug was administered at a dose of $200 \mathrm{mcg}$ vaginally, from 20 weeks of gestation. Of the 43 pregnancies studied, 20 were treated with progesterone. There was no association between use of progesterone and delivery time, so that the use of natural micronized progesterone did not prevent preterm birth $(p=0.87)$. Therefore, natural micronized progesterone did not reduce the frequency of preterm birth in twin pregnancies.

Keywords: Twin pregnancy; progesterone

A incidência de gestações gemelares sofreu uma explosão nas últimas décadas, reflexo do crescimento do uso das técnicas de reprodução assistida. As gestações múltiplas correspondem a 2 a $3 \%$ de todas as gravidezes e vêm ganhando importância devido ao aumento de sua prevalência e ao risco elevado de complicações materno-fetais, especialmente de parto prematuro ${ }^{1-3}$.

Apesar de todos os esforços e avanços da medicina perinatal, a taxa de mortalidade nas gestações múltiplas permanece mais alta do que a observada em gestações únicas ${ }^{2}$. Sendo assim, um desafio da prática obstétrica é prevenir a prematuridade e, para tal, várias estratégias estão sendo adotadas, como o uso profilático da progesterona exógena ${ }^{4-6}$. 
A progesterona é um hormônio sintetizado pelos ovários, corpo lúteo, placenta e adrenais que tem como função a manutenção da gestação ao promover o relaxamento da musculatura lisa e atuar sobre o amadurecimento cervical, diminuindo a ação uterotônica da ocitocina, alterando a expressão dos receptores de estrogênio no miométrio e potencializando a ação dos beta-adrenérgicos nos receptores uterinos. A progesterona natural micronizada tem propriedades similares às da natural como, por exemplo, efeitos gestagênico, antiestrogênico, antiandrogênico leve e antialdosterona. Entretanto, como explicar a ação da progesterona exógena em uma paciente grávida, com elevação fisiológica da progesterona endógena? A explicação está associada à modulação de receptores responsáveis pelo desencadeamento do trabalho de parto prematuro (TPP) de origem inflamatória e/ou infecciosa ${ }^{7-10}$.

As evidências científicas apoiam o uso da progesterona na redução do parto pré-termo ${ }^{2}$. Entretanto, para o uso em gestações gemelares, os resultados não têm sido animadores. Parece-nos importante avaliar a resposta à progesterona em nossa população, já que muitos resultados não podem ser extrapolados, pois muitos estudos avaliaram a resposta ao caproato de progesterona, injetável e intramuscular, e outros à progesterona natural micronizada em gestantes com outros fatores de risco para TPP, como, por exemplo, o colo curto ${ }^{1,6,11}$. Para tanto, nos propomos avaliar a eficácia do uso de progesterona natural micronizada, administrada via vaginal, na prevenção do parto prematuro em gestações gemelares.

\section{MÉTODOS}

O Serviço de Alto Risco da Universidade Federal de Juiz de Fora e o Serviço de Obstetrícia da Faculdade de Medicina de Barbacena atendem pacientes encaminhadas da própria instituição ou de outros serviços. Os serviços prestam atendimento à população de baixa renda e utilizam o mesmo prontuário padrão. Foram incluídas pacientes com gestações gemelares, independentemente da sua zigoticidade e corionicidade. Gestações únicas e pacientes com indicação obstétrica para interrupção prematura da gravidez gemelar foram excluídas.

Trata-se de uma coorte histórica. Selecionaram-se as gestações gemelares que foram atendidas no período de janeiro de 2008 a janeiro de 2015. Identificaram-se 52 pacientes com gestações gemelares. Com a exclusão daquelas que abandonaram o pré-natal ou evoluíram para abortamento, foram avaliadas neste estudo 43 pacientes. Como desfecho principal considerou-se o parto prematuro, e como desfechos secundários a internação para inibição do trabalho de parto e infecção urinária.

A decisão sobre o uso da medicação foi baseada no aceite da paciente, conforme preconiza o Código de Ética Médica (artigo XXI) ${ }^{12}$, que orienta ao médico aceitar as escolhas de seus pacientes relativas aos procedimentos diagnósticos e terapêuticos, desde que adequadas ao caso e reconhecidas cientificamente. As pacientes foram divididas em dois grupos: expostas, composto por gestantes que receberam progesterona natural micronizada (Grupo $1 ; n=20$ ) e não expostas, composto por gestantes que não concordaram em usar a medicação (Grupo 2; n=23). A dosagem foi de $200 \mathrm{mcg}$ por dia, via vaginal, a partir de 20 semanas de gestação até o limite de 33 semanas e 6 dias, por no mínimo 4 semanas, ou na primeira consulta de pré-natal, quando esta ocorreu acima de 20 semanas.

Os dados foram digitados e armazenados no software Epi-Info, versão 8.0. Calcularam-se os valores de risco relativo $(R R)$ em tabelas $2 \times 2$. A regressão logística foi usada para avaliar a independência dos efeitos observados. Foi considerado como nível de significância $p<0,05$. O estudo foi aprovado pela Comissão de Ética da Fundação Hospitalar do Estado de Minas Gerais e Faculdade de Medicina de Barbacena.

\section{RESULTADOS}

Das pacientes estudadas, $20(46,51 \%)$ usaram a progesterona natural micronizada. Os dados clínicos e epidemiológicos das pacientes são apresentados na Tabela 1.

A frequência de TPP foi de $48,8 \%(n=21)$, com média em idade gestacional de $33,02 \pm 3,6$ semanas de gestação, sendo a mínima com 24 semanas e a máxima com 39 semanas de gestação. Não houve associação entre época do parto e uso da progesterona ( $p=0,8 ; R R=1,04 ; \mathrm{IC} 95 \%$ 0,57-1,8).

Quando as pacientes foram estratificadas por idade gestacional, também não houve diferença entre gestações abaixo de 28 semanas $(p=0,94$; $\mathrm{RR}=0,95$ ); intervalo de confiança de $95 \%$ (IC95\% $0,87-1,04)$, abaixo de 34 semanas $(p=0.5 ; R R=1,18$; IC95\% 0,8-1,6) e abaixo de 37 semanas ( $p=0,8$; $\mathrm{RR}=1,0$; IC95\% 0,58-1,8). Embora as usuárias de progesterona tenham iniciado o pré-natal mais precocemente, não houve associação entre o uso da progesterona e TPP $(p=0,8)$. A associação entre tocólise e parto prematuro não foi significativa. Das oito pacientes que foram submetidas à tocólise, seis eram usuárias de progesterona; destas, cinco tiveram êxito na inibição, não evoluindo para TPP. 
Tabela 1: Dados clínicos e epidemiológicos das pacientes avaliadas e associação com o uso da progesterona natural micronizada.

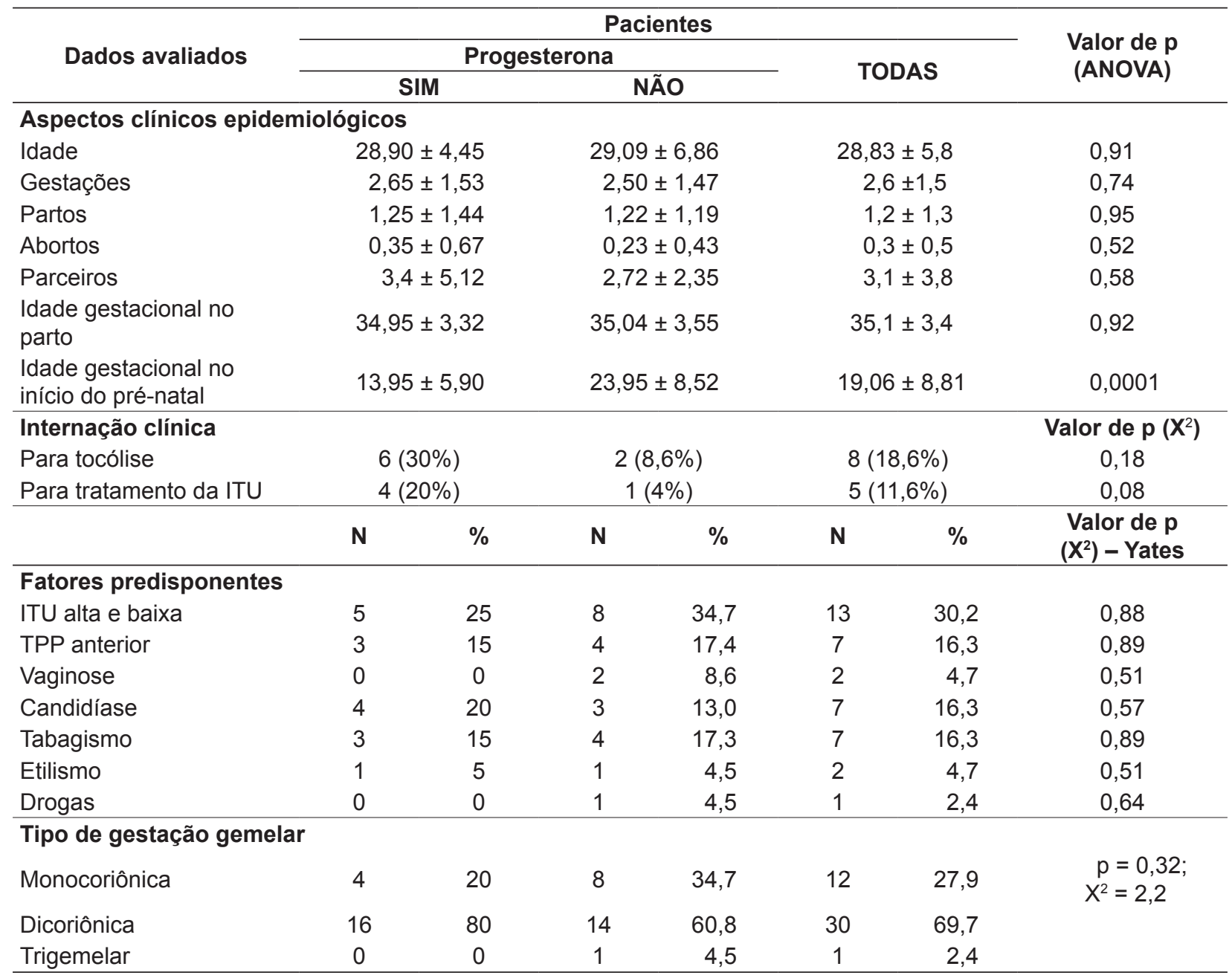

ITU = infecção do trato urinário; TPP = trabalho de parto prematuro.

Entretanto, as duas pacientes não usuárias de progesterona evoluíram para TPP (tabela 2)

A associação entre progesterona e internação para inibição do TPP não foi significativa $(R R=1,3$; $p=0,16$; IC95\% 0,95-1,78), de forma que o uso da progesterona natural micronizada não impediu o desencadeamento do TPP.

Quando se comparou uso da progesterona natural micronizada e infecção urinária $(n=13)$, verificou-se que 5 cinco $(11,6 \%)$ apresentaram infecção urinária alta e foram internadas para tratamento da infecção urinária e inibição do TPP.

\section{DISCUSSÃO}

A frequência de gestação gemelar é de 1 a $2 \%$ na população geral ${ }^{1}$. Existem serviços que atendem pacientes de fertilização in vitro onde a taxa de gestação múltipla pode chegar a $25 \%$. Esse não é o caso dos serviços analisados neste estudo.
A maioria das gestações múltiplas acompanhadas foi natural, compatível com a frequência relatada na literatura médica ${ }^{1,13}$.

A média de idade gestacional na época do parto foi de 35 semanas. A progesterona natural micronizada, quando administrada por via vaginal, não se mostrou benéfica na profilaxia do TPP, visto que a frequência desse evento foi semelhante nos dois grupos. Tais resultados são compatíveis com os dados de Hartikainen-Sorri et al. ${ }^{11}$, Rouse et al. ${ }^{14}$, e Lim et al. ${ }^{15}$. Além disso, Combs et al. ${ }^{16}$, que utilizaram $250 \mathrm{mg}$ de caproato de progesterona injetável semanal, não verificaram redução da taxa de prematuridade. No Brasil, a via vaginal foi difundida desde a publicação dos estudos de Fonseca et al. ${ }^{2,6-7}$, que fizeram uso da progesterona natural micronizada por via vaginal, na dosagem de $100 \mathrm{mcg}$ a partir de 24 semanas, em gestações únicas. Entretanto, em subanálise realizada com 24 gestantes gemelares 
Tabela 2: Frequência de trabalho de parto prematuro e associação com o uso da progesterona natural micronizada.

\begin{tabular}{|c|c|c|c|c|c|c|}
\hline \multirow{2}{*}{\multicolumn{2}{|c|}{ Variáveis }} & \multicolumn{2}{|c|}{ Progesterona } & & \multirow{2}{*}{$\mathbf{P}$} & \multirow{2}{*}{$X^{2}$ (Yates) } \\
\hline & & SIM & NÃO & & & \\
\hline \multirow{3}{*}{$\begin{array}{l}\text { Trabalho de parto } \\
\text { prematuro }\end{array}$} & SIM & 10 & 11 & 21 & \multirow{3}{*}{0,87} & \multirow{3}{*}{0,02} \\
\hline & NÃO & 10 & 12 & 22 & & \\
\hline & Total & 20 & 23 & 43 & & \\
\hline \multirow{3}{*}{$\begin{array}{l}\text { Internação para } \\
\text { tocólise }\end{array}$} & SIM & 6 & 2 & 8 & \multirow{3}{*}{0,16} & \multirow{3}{*}{1,9} \\
\hline & NÃO & 14 & 21 & 35 & & \\
\hline & Total & 20 & 23 & 43 & & \\
\hline \multirow{3}{*}{ Exxito na tocólise } & SIM & 5 & 2 & 7 & \multirow{3}{*}{0,38} & \multirow{3}{*}{0,53} \\
\hline & NÃO & 1 & 0 & 1 & & \\
\hline & Total & 6 & 2 & 8 & & \\
\hline \multirow{3}{*}{ Infecção urinária } & SIM & 5 & 8 & 13 & \multirow{3}{*}{0,7} & \multirow{3}{*}{0,13} \\
\hline & NÃO & 15 & 15 & 30 & & \\
\hline & Total & 20 & 23 & 43 & & \\
\hline
\end{tabular}

com colo curto, a progesterona natural não mostrou a mesma eficácia ${ }^{2,6,7}$.

O estudo Progesterone for the prevention of preterm birth in twin pregnancy (STOPPIT) $)^{17}$ randomizou pacientes para a progesterona vaginal diária (gel de $90 \mathrm{mg} ; \mathrm{n}=250)$ ou gel placebo $(\mathrm{n}=250)$ durante 10 semanas a partir de 24 semanas de gestação. A proporção de morte intrauterina ou parto antes de 34 semanas de gravidez foi de $24,7 \%$ no grupo de progesterona e $19,4 \%$ no grupo placebo $(O R=1,36$; IC95\% 0,89-2,09; $p=0,16)$, confirmando que a progesterona não impediu o nascimento prematuro gemelar (IC95\% 0,89-1,51). Klein et al. ${ }^{18}$ [PRospective Evaluation of Diabetic Ischemic heart disease by Computed Tomography (PREDICT) study], ao analisarem gestantes gemelares entre 20 a 34 semanas, não identificaram diferenças quanto à época do parto entre pacientes com colo curto $(p=0,87)$ ou com história de parto prematuro $(p=0,62)$. Brubaker et al. ${ }^{19}$, em estudo com 167 gestações gemelares, trataram 61 (35,7\%) com progesterona vaginal. A taxa de risco para parto antes de 35 semanas de gestação no grupo com progesterona vaginal foi de 1,8 (IC95\% 1,5-3,1). Outro estudo avaliou o uso da progesterona natural em gestações gemelares diamnióticas e dicoriônicas, com o dobro da dose habitualmente utilizada na gestação única (400 mg/dia), mas os resultados não foram animadores ${ }^{20}$.

Apesar de nossa amostragem ser pequena, a frequência de gestação gemelar reflete aquela identificada na literatura para serviços que não trabalham com fertilização assistida ${ }^{1}$. Nossos resultados apoiam estudos internacionais que afirmam que, até o momento, a profilaxia do TPP com progesterona natural micronizada não apresenta benefício em gestações gemelares. Deste modo, ainda não existe, até o momento, intervenção que reduza a chance de prematuridade na gestação gemelar. Por isso, pode-se afirmar que é necessário adquirir novos conhecimentos sobre a etiologia e a fisiopatologia do parto pré-termo, afastando fatores de risco para a prematuridade, e oferecer um pré-natal de qualidade, minimizando os riscos para mãe e bebê ${ }^{21}$.

\section{REFERÊNCIAS}

1. Zork N, Biggio J, Tita A, Rouse D, Gyamfi-Bannerman C. Decreasing prematurity in twin gestations: predicaments and possibilities. Obstet Gynecol. 2013;122(2 Pt 1):375-9. http://dx.doi.org/10.1097/ AOG.0b013e31829b2dba. PMid:23969808.

2. Fonseca EB, Bittar RE, Carvalho $\mathrm{MH}$, Zugaib M. Prophylactic administration of progesterone by vaginal suppository to reduce the incidence of spontaneous preterm birth in women at increased risk: a randomized placebo-controlled double-blind study. Am J Obstet Gynecol. 2003;188(2):419-24. http:// dx.doi.org/10.1067/mob.2003.41. PMid:12592250.

3. Goldenberg RL, Culhane JF, lams JD, Romero R. Epidemiology and causes of preterm birth. Lancet. 2008;371(9606):75-84. http://dx.doi. org/10.1016/S0140-6736(08)60074-4. PMid:18177778.
4. Kosinska-Kaczynska K, Szymusik I, Bomba-Opon D, Madej A, Oleszczuk J, Patro-Malysza J, et al. Late prematurity in twins: a Polish multicenter study. Twin Res Hum Genet. 2014;17(5):369-75.

5. Kazemier BM, Buijs PE, Mignini L, Limpens J, Groot CJ, Mol BW, et al. Impact of obstetric history on the risk of spontaneous preterm birth in singleton and multiple pregnancies: a systematic review. BJOG. 2014;121(10):1197-208. http://dx.doi. 
org/10.1111/1471-0528.12896. PMid:24899245.

6. Fonseca EB, Bittar RE, Tosta K, Zugaib M. Progesterona e prevenção do parto prematuro: aspectos atuais. Femina. 2008;36(12):771-7.

7. Fonseca EB, Bittar RE, Damião R, Zugaib M. Prematurity prevention: the role of progesterone. Curr Opin Obstet Gynecol. 2009;21(2):1427. http://dx.doi.org/10.1097/ GCO.0b013e3283294770. PMid:19300251.

8. Lang CT, lams JD. Goals and strategies for prevention of preterm birth: an obstetric perspective. Pediatr Clin North Am. 2009;56(3):53763. http://dx.doi.org/10.1016/j. pcl.2009.03.006. PMid:19501691.

9. Xu H, Gonzalez JM, Ofori E, Elovitz MA. Preventing cervical ripening: the primary mechanism by which progestational agents prevent preterm birth? Am J Obstet Gynecol. 2008;198(3):314.e1-8. http://dx.doi. org/10.1016/j.ajog.2008.01.029. PMid:18313454.

10. Yoshizaki CT, Bittar RE, Francisco RPV, Zugaib M. Progesterona para prevenção do parto prematuro. Femina. 2013;40(2):79-86.

11. Hartikainen-Sorri AL, Kauppila A, Tuimala R. Inefficacy of 17 alphahydroxyprogesterone caproate in the prevention of prematurity in twin pregnancy. Obstet Gynecol. 1980;56(6):692-5. PMid:7443111.

12. Conselho Federal de Medicina (CFM). Código de Ética Médica: confiança para o médico, segurança para o paciente. CFM; 2015. [citado 2015 ago 15]. Disponível em: http://www. portalmedico.org.br/novocodigo/ integra.asp

13. Amorim AMA, Silva LR, Rosado LEP. Eficácia da progesterona na prevenção do trabalho de parto prematuro gemelar: revisão sistemática. Femina. 2015;43(1):1320.

14. Rouse DJ, Caritis SN, Peaceman AM, Sciscione A, Thom EA, Spong $\mathrm{CY}$, et al. A trial of 17 alphahydroxyprogesterone caproate to prevent prematurity in twins. $N$ Engl J Med. 2007;357(5):454-61. http:// dx.doi.org/10.1056/NEJMoa070641. PMid:17671253.

15. Lim AC, Schuit E, Bloemenkamp K, Bernardus RE, Duvekot JJ, Erwich JJ, et al. 17a -hydroxyprogesterone caproate for the prevention of adverse neonatal outcome in multiple pregnancies: a randomized controlled trial. Obstet Gynecol. 2011;118(3):51320. http://dx.doi.org/10.1097/ AOG.0b013e31822ad6aa. PMid:21860279.

16. Combs CA, Garite T, Maurel K, Das A, Porto M, Obstetrix Collaborative Research Network. 17-hydroxyprogesterone caproate for twin pregnancy: a double-blind, randomized clinical trial. Am J Obstet Gynecol. 2011;204(3):221.e1-8. http:// dx.doi.org/10.1016/j.ajog.2010.12.042. PMid:21376161.

17. Norman JE, Mackenzie F, Owen $\mathrm{P}$, Mactier H, Hanretty K, Cooper $\mathrm{S}$, et al. Progesterone for the prevention of preterm birth in twin pregnancy (STOPPIT): a randomised, double-blind, placebo-controlled study and meta-analysis. Lancet. 2009;373(9680):2034-40. http://dx.doi. org/10.1016/S0140-6736(09)60947-8. PMid:19523680.

18. Klein K, Rode L, Nicolaides KH, Krampl-Bettelheim E, Tabor A, PREDICT Group. Vaginal micronized progesterone and risk of preterm delivery in high-risk twin pregnancies: secondary analysis of a placebocontrolled randomized trial and meta-analysis. Ultrasound Obstet Gynecol. 2011;38(3):281-7. http:// dx.doi.org/10.1002/uog.9092. PMid:21739499.

19. Brubaker SG, Pessel C, Zork N, Gyamfi-Bannerman C, Ananth CV. Vaginal progesterone in women with twin gestations complicated by short cervix: a retrospective cohort study. BJOG. 2015;122(5):712-8. http:// dx.doi.org/10.1111/1471-0528.13188. PMid:25428801.

20. Serra V, Perales A, Meseguer J, Parrilla JJ, Lara C, Bellver $\mathrm{J}$, et al. Increased doses of vaginal progesterone for the prevention of preterm birth in twin pregnancies: a randomised controlled double-blind multicentre trial. BJOG. 2012;120(1):50-7. http://dx.doi.org/10.1111/j.14710528.2012.03448.x. PMid:22882759.

21. Bittar RE, Francisco RPV, Zugaib M. Prematuridade: quando é possível evitar? Rev Bras Ginecol Obstet. 2013;35(10):4335. http://dx.doi.org/10.1590/ S0100-72032013001000001. PMid:24337053. 